Rev. Elev. Méd. vét. Pays trop., 1974, 27 (4) : 437-450

\title{
Appréciation comparée de la densité d'une population isolée de Glossina tachinoides West. par deux méthodes classiques : le circuit de capture et les marquages-recaptures
}

\author{
par D. CUISANCE (*)
}

\begin{abstract}
RESUME
Des méthodes d'évaluation de la densité apparente (méthode du «circuit de capture » ou « fly-round ») et de la densité réelle (marquagerecapture simple ou répété) ont été appliquées simultanément dans un gîte à Glossina tachinoides durant la saison sèche, période climatique relativement stable, au cours de laquelle les glossines se concentrent dans un gîte riverain du Chari.

L'évolution qualitative des courbes de densité de la population ainsi isolée est semblable avec les deux méthodes dans les conditions de l'observation.

Quantitativement, seules les méthodes de marquage-recapture permettent une évaluation de la densité réelle; l'approche de celle-ci par ces méthodes dépend de leur régularité d'application, des délais de marquages ou de recaptures; clles nécessitent aussi des populations numériquement importantes sous peine de perdre leur validité. La méthode du \& circuit de capture » peut alors leur être substituée et rendre de grands services.
\end{abstract}

\section{INTRODUCTION}

Le dénombrement exact de la totalité des individus d'une espèce donnée, dans une aire géographique même limitée, est d'une réalisation généralement difficile, voire impossible. $\mathrm{Si}$ des mesures directes sont inaccessibles, des méthodes d'appréciation indirecte sont utilisables et permettent, malgré les erreurs qui leur sont inhérentes, de rendre de grands services.

La lutte contre les insectes vecteurs de germes pathogènes continue à susciter un vif intérêt; c'est précisément le cas des glossines; pour juger de l'efficacité des méthodes mises en cuvre, il est indispensable de pouvoir appré-

(*) I.E.M.V.T., Région de Recherches vétérinaires et zootechniques de l'Afrique Centrale, Laboratoire de Farcha, B.P. 433, N'Djamena, Tchad. cier la densité d'une population avant, pendant et après une intervention. Des investigations semblables s'imposent de même lorsqu'il s'agit de suivre la dynamique d'une population sous l'effet des conditions climatiques ou de comparer des variations saisonnières ou annuelles de densité.

Le but de la présente note est de signaler les résultats enregistrés avec deux méthodes d'étude classiques, utilisées simultanément dans un gîte à Glossina tachinoides, dont la population isolée subit de grandes variations de densité durant la saison sèche.

\section{HISTORIQUE}

Les premiers observateurs s'étaient vite aperçus que les glossines étaient confinées à des 
zones de végétation assez bien définies (fly-belt) et qu'il suffisait de parcourir ces lieux à des périodes précises pour capturer des mouches.

FISKE (4), citant déjà quelques auteurs l'ayant précédé, donne les premiers résultats des échantillonnages obtenus par cette méthode. Les entomologistes anglophones d'Afrique de l'Est et de l'Ouest adoptent cette méthode appelée Fly-round; cette technique du circuit de capture fut vraiment définie en 1930 par POTTS (20) comme étant l'étude d'une zone à travers laquelle l'entomologiste trace un sentier traversant différents types de végétation; l'équipe de captureurs la parcourt à des intervalles réguliers pendant une longue période, récoltant sur chaque tronçon du circuit toutes les glossines et notant l'espèce, le sexe et un grand nombre d'autres renseignements. Utilisée par de nombreux entomologistes de terrain, cette méthode a fourni des données importantes, variables suivant les espèces de glossines, et dont les résultats ont été comparés avec la méthode de marquage-recapture [JACKSON (12), GLASGOW \& DUFFY (8), GLASGOW \& WELCH (9)]. Cette dernière, plus précise, est généralement adoptée dans l'étude des densités de population.

Les premières données sur les proportions d'animaux marqués recapturés semblent avoir été fournies par PETERSEN (3) sur les poissons. Mais c'est LINCOLN (17) travaillant sur des canards en Amérique du Nord, qui donne une formule d'estimation d'une population appelée "Index de Lincoln " (nombre d'animaux marqués relachés, divisé par la proportion trouvée marquée lors de la recapture). Sur les glossines, JACKSON (11), dans l'Est Africain, appliqua cet indice et le perfectionna (13) dans ses méthodes dites " positive et négative ".

On citera les travaux de DOWDESWELL, FISCHER et FORD sur les Lépidoptères, ceux de' GILMOUR, WATERHOUSE et Mc INTYRE sur la mouche bleue du mouton, ceux de LAMOTTE sur les escargots, SLUITER, VAN HEERDT et BEZEM sur les chauvessouris, AGUESSE sur les libellules (15).

Des traitements mathématiques très élaborés essayant de cerner au maximum la densité réelle d'une population et ses fluctuations ont été entrepris par LESLIE et CHITTY (16), BAILEY $(2,3)$ entre autres.
Ces analyses très complexes, susceptibles de rendre de grands services, sont d'un maniement difficile et restent, malgré tout, entachées d'erreurs, car tous les facteurs conditionnant la dynamique de la population analysée ne peuvent être pris en considération.

Parce qu'il convenait de perturber le moins possible la population étudiée, mais qu'il fallait en extraire le plus grand nombre de données possibles, tout en utilisant des moyens simples d'investigations, nous avons retenu comme méthode de base celle du marquagerecapture simple ou répété, appliquée de façon hebdomadaire dans un gîte donné dont il convenait de suivre la dynamique de population. La constitution du lot de mouches à marquer se réalise au cours d'un circuit de capture dans le gîte ou fly-round; la recapture, à un délai donné, a lieu suivant le même protocole. On est ainsi amené à comparer un nombre brut de glossines capturées dans un nombre d'heures fixes par rapport aux résultats obtenus par la méthode du marquage-recapture, sensée fournir une densité réelle et à se demander si les deux types de résultats concernant $G$. tachinoides sont liés entre eux au cours de la saison d'observation.

\section{LIEUX D'OBSERVATION}

Décrit dans une précédente publication (5), le gîte retenu est situé dans la réserve de faune de la Kalamaloué, à une quinzaine de kilomètres de N'Djamena, sur la rive camerounaise du Chari; il a l'aspect d'une bande étroite de végétation $(1000 \times 100 \mathrm{~m})$, très dense, en forme de fuseau, tapissant des bas-fonds argilosableux, sans eau au cours de la saison sèche, mais totalement inondés en période de crue du Chari ou de ses défluents en saison des pluies. La population de glossines (Glossina tachinoides uniquement) ne s'installe qu'après le retrait des eaux d'inondation en saison sèche et fraîche (décembre).

En saison des pluies, les glossines sont fortement dispersées du fait des conditions climatiques favorables à cette époque, mais avec la sécheresse et les fortes températures, elles se regroupent et se concentrent dans ces zones végétales que l'on appelle gîtes.

Le gîte choisi reste parfaitement isolé, car entouré de grandes savanes arides qui consti- 
tuent des barrières infranchissables pour les glossines à ce moment de l'année. Il faut donc souligner qu'il s'agit là d'une population isolée dans l'espace et que l'observation, qui a lieu de février à juin, s'étale sur une période climatique relativement homogène, caractérisée par de fortes chaleurs et une humidité basse, mais qui tend à s'élever à mesure que l'on se rapproche de la saison des pluies (juillet).

\section{METHODE DU CIRCUIT DE CAPTURE OU “ FLY-ROUND »}

Le nombre de mouches capturées dépend de la densité de la population, mais aussi de l'activité des insectes. Aussi, certains auteurs parlent-ils de "densité-activité " obtenue par cette méthode. NASH (4) employa le terme de densité apparente par rapport à la densité réelle obtenue par la méthode de marquage-recapture.

\section{A. Protocole expérimental}

Les auteurs anglais distinguent au moins quatre types de "fly-round», les captures se faisant soit en se déplaçant, soit à des points fixes.

- Le circuit commun : long de 5 miles, il est parcouru par l'équipe qui s'arrête tous les 50 ou 100 yards pour capturer les mouches.

- Le vegetation fly-round: composé de plusieurs sections qui ont trait à différents types de végétation, il est utilisé pour établir des rapports entre la concentration en glossines et les groupements végétaux.

- Le transect fly-round: le parcours est droit et traverse la zone à étudier, qui est divisée en sections au bout de chacune desquelles ont lieu les captures; généralement, plusieurs parcours parallèles entre eux sont définis.

- Le picket fly-round : on choisit 6 à 8 places de capture, où les captureurs stationnent une heure environ; c'est une capture à points fixes.

Dans notre observation, il s'agit d'un circuit de type commun se rapprochant cependant fortement du vegetation fly-round. L'équipe de captureurs (7-8 membres) reste groupée dans un rayon de 20 à $30 \mathrm{~m}$ autour du chef d'équipe et se déplace dans le gîte en s'arrêtant tous les
$25 \mathrm{~m}$ environ; le circuit, qui dans sa forme (un huit) reste toujours le même, est parcouru deux fois dans la journée et traverse les zones de végétation connues pour être propices aux glossines.

Les heures de capture sont imposées principalement par les facteurs thermiques, hygrométriques, photoniques, mais la température ambiante conditionne à elle seule en grande partie l'activité de Glossina tachinoides dont l'optimum se situe entre $30-32^{\circ}$ : en saison chaude les captures ont lieu le matin entre $7 \mathrm{~h} 30$ et $11 \mathrm{~h}$. Enfin cette méthode s'applique d'autant mieux à $G$. tachinoides que celle-ci pique préférentiellement l'homme (espèce anthropophile). Le captureur, qui représente l'appât, prend à l'aide d'un filet la glossine qui veut le piquer ("capture par unité d'effort" de type attractif). Le but est donc de donner à toute la population du gîte l'occasion de piquer l'homme et donc d'être capturée, afin de collecter le plus grand nombre de glossines par jour, suivant un protocole régulièrement constant.

\section{B. Résultats}

GRUVEL (10), travaillant sur plusieurs gîtes à $G$. tachinoides, exprime la densité apparente par la moyenne horaire des captures faites dans une journée ou activité moyenne journalière horaire (A.M.J.H.). Pour notre part, durant les mois de février, mars, avril, mai, la méthode du circuit de capture a été pratiquée deux fois par semaine, et le nombre de glossines capturées est rapporté au nombre d'heures de capture et au nombre de captureurs, ce qui permet d'obtenir le nombre de glossines/heure/captureur. Une moyenne est faite des deux résultats obtenus chaque semaine et cette donnée moyenne est portée sur le graphique I (p. 440). Il est bien connu que, lors de captures au filet, le pourcentage des sexes est très différent : on capture environ $2 / 3$ de mâles pour $1 / 3$ de femelles, à cette période de l'année; ces dernières sont donc très mal représentées dans ce type d'échantillonnage. On retiendra seulement la courbe obtenue pour le total mâles et femelles et celle résultant des mâles seuls, les données chiffrées pour les femelles étant trop faibles. L'étude repose sur 20920 glossines capturées dont 14367 mâles.

On observe alors un accroissement rapide de la population; elle atteint son maximum à la 


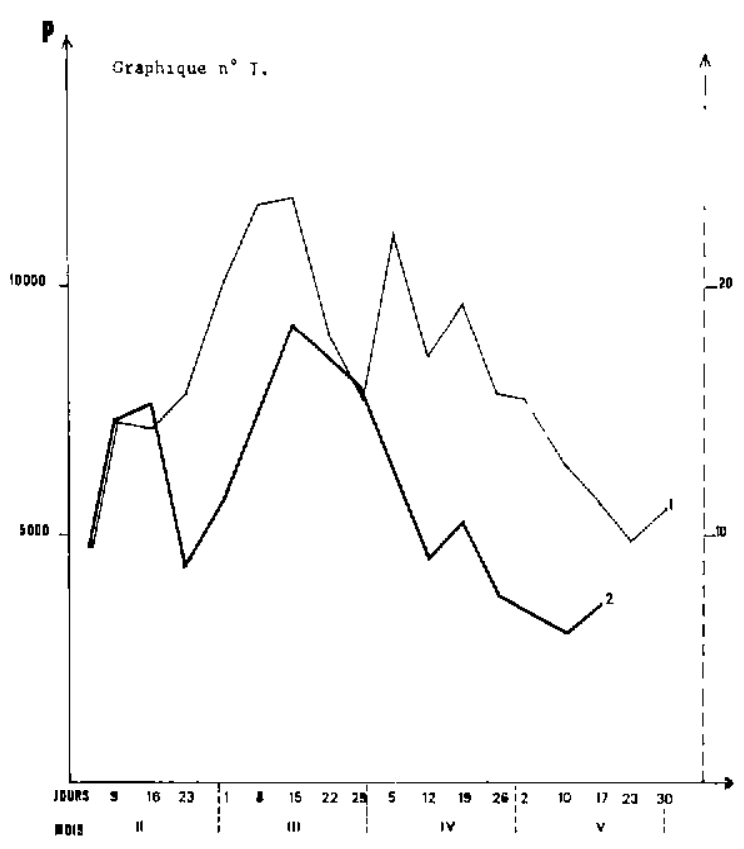

Graph. $n^{o}$ I. - Evolution de la densité de population (mâles et femelles) suivant la méthode du circuit de capture (1) et la méthode du marquage-recapture (2).

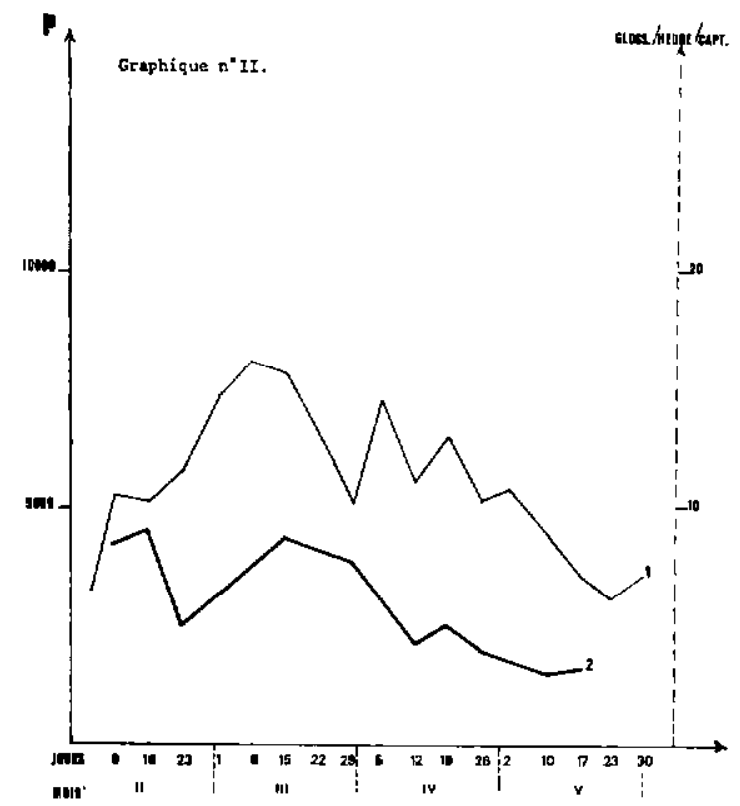

Graph. $\mathbf{n}^{\circ}$ II. - Evolution de la densité de population (mâles seulement) suivant la méthode du circuit de capture (1) et la méthode du marquage-recapture (2).

mi-mars, puis décroît au cours de la fin mars ainsi qu'en avril et mai; la décroissance apparaît en dents de scie sur le graphique.
L'allure générale obtenue à partir des captures totales (mâles et femelles) est donc celle d'une courbe en clocher, celui-ci se situant à la mi-mars.

Pour la population de mâles seuls (graphique II) le même type de courbe apparaît; après une croissance rapide de la densité de population, on atteint le sommet du clocher qui se situe cette fois-ci 8 jours plus tôt par rapport à la courbe précédente, puis une décroissance en dents de scie lui fait suite au cours des mois d'avril et mai.

\section{METHODE \\ DU MARQUAGE-RECAPTURE SIMPLE}

Elle permet d'obtenir théoriquement l'effectif réel de la population et donc d'en suivre les fluctuations. Si la méthode précédente n'apporte qu'une information relative, celle-ci est sensée donner un résultat quantitativement précis.

Dans différents gîtes de la réserve de la Kalamaloué, cette méthode a été employée entre 1966 et 1970 par GRUVEL (10) qui a pratiqué des sondages à des intervalles variables et en retenant des délais de recaptures plus ou moins grands, dans le but d'obtenir diverses données écologiques.

Nous avons repris la méthode en l'appliquant régulièrement toutes les semaines sur une période de quatre mois dans un gîte défini et en respectant un protocole rigoureusement constant de capture, de marquage et de recapture afin d'obtenir la courbe évolutive de la densité de population.

\section{A. Protocole expérimental}

Le principe est simple et n'est que la transposition de la méthode de dilution d'une substance colorée employée pour évaluer le volume d'un liquide qu'on ne peut recueillir en son entier (Le Berre).

$$
\mathbf{P}=\frac{\text { M.T. }}{\mathrm{m}}
$$

$\mathbf{P}=$ Population estimée.

$\mathrm{M}=\mathrm{N}^{\text {bre }}$ de mouches marquées lâchées.

$\mathrm{T}=\mathrm{N}^{\text {bre }}$ de mouches capturées.

$\mathrm{m}=\mathrm{N}^{\text {bre }}$ de mouches marquées recapturées. 
Cette méthode ne s'applique que si $\mathrm{M}, \mathrm{T}$ et $\mathbf{m}$ sont assez grands et l'erreur standard calculée est :

$$
\sigma=\sqrt{\frac{\mathrm{m}(\mathrm{T}-\mathrm{m})}{\mathrm{T}^{3}}}
$$

ce qui permet d'apprécier les valeurs supérieure et inférieure probables de $P$ au risque choisi de 5 p. 100.

Entre le marquage et la recapture, un délai de 48 heures a été retenu; tout en permettant aux glossines marquées de se "diluer" dans la population, il n'autorise pas de grandes variations dans la natalité et la mortalité de celle-ci. Une étude sur la dispersion des glossines (vitesse et étendue) dans le gîte (5) a montré qu'au bout de 48 heures, la dispersion de mouches marquées pouvait être considérée comme très satisfaisante.

Comme il a été dit précédemment, la population du gîte décrit est isolée dans l'espace; aucune émigration ou immigration ne peuvent donc faire fluctuer une telle population. Des mouches marquées dans d'autres gîtes n'ont jamais été retrouvées dans ce gîte au cours de la saison chaude, alors qu'elles le sont facilement en saison des pluies; de même, aucune mouche marquée dans le gîte n'a été retrouvée dans les gites voisins. En dernier lieu, aucune perturbation profonde n'est venue modifier brutalement la population (abattage d'arbres, suppression des hôtes nourriciers constitués principalement par les guibs, feux de brousse, passage de troupeaux, etc.). Situé en pleine réserve, le biotope a été respecté. Les résultats obtenus dans ces conditions peuvent donc être considérés comme valables.

La technique de marquage utilisée est simple, rapide, pratique. Elle consiste à pulvériser sur les mouches, introduites par groupes d'une dizaine dans un tube à essai, un jet de poudre fluorescente (SWITZER Brothers Ins.) (23), ou à déposer avec un stylet sur le thorax de l'insecte une petite goutte de gouache (LINEL 7-77; J. M. PAILLARD). Ces marques restent visibles, sans toutefois trop attirer l'attention des prédateurs (lézards, araignées) ni affecter la vie des individus.

Les mouches marquées ont été libérées en un seul point (01) pour les lâchers, sauf ceux du 16 et 23 février, qui ont eu lieu en un autre point (02) distant de $250 \mathrm{~m}$.
Durant la même période que précédemment (février, mars, avril, mai), la méthode de capture-marquage simple est pratiquée régulièrement chaque semaine; la couleur du marquage change à chaque sondage.

\section{B. Résultats}

Le total des glossines marquées et lâchées (M) correspond au total de celles récoltées lors d'un circuit de capture de cinq heures trente; la recapture $(T)$, quarante huit heures après, a lieu suivant le même circuit. Les valeurs de la population $\mathbf{P}$ ainsi estimée, sont portées sur le graphique I, qui traduit l'évolution de cette dernière dans le temps d'observation considéré.

La courbe obtenue avec les chiffres globaux (mâles et femelles) indique une baisse de population entre le 16 et le 23 février, puis une croissance rapide jusqu'à la mi-mars suivie d'une décroissance en dents de scie durant avril et mai.

Si l'on considère la population $\mathbf{P}$ rapportée aux seules captures de mâles (graphique II), on observe une courbe qui suit la même évolution et pour laquelle le maximum est également atteint le 15 mars.

\section{COMPARAISON DES COURBES DE POPULATION OBTENUES PAR LES DEUX METHODES}

- Sur le graphique I où sont portées les courbes évolutives obtenues par les deux méthodes sur l'ensemble des mouches, sans tenir compte des sexes, l'aspect en clocher est le même, les maximums étant atteints à la mème date (15 mars). Les données fournies par les deux méthodes aboutissent donc à des courbes de population d'aspect général semblable. On notera simplement la différence mineure suivante : entre le 16 et le 23 février, la courbe obtenue par la méthode de marquage-recapture accuse une chute, qui trouve une explication dans le fait que pour ces deux dates, les lâchers de glossines marquées ont eu lieu en un point (02), qui n'est pas celui habituellement utilisé pour tous les autres lâchers (01); or, les conditions de survie ne sont pas les mêmes en ces deux points, 02 étant moins bien abrité que 01 , ce qui donne des valeurs de $m$ différentes, et explique ce décrochement dans la courbe. 
- Sur le graphique II qui a trait aux courbes obtenues à partir des seules captures de mâles, on notera l'identité d'évolution avec la même réserve pour la période du 16 au 23 février. Toutefois, il faut signaler que les maximums pour les deux courbes accusent un léger décalage de 8 jours, qui reste une différence minime.

- D'une façon générale, on obtient par les deux méthodes utilisées dans les conditions définies (isolement de la population, stabilité relative des conditions climatiques à cette saison) des résultats très comparables.

- Avec la méthode du circuit de capture, JACKSON (12) travaillant sur Glossina morsitans, aboutit à des conclusions identiques; il considère que "les captures de glossines sur des circuits réguliers offrent une mesure sérieuse des changements de densité réelle» d'une population. Il sous-estime même fortement le facteur activité, qu'il considère comme mineur.

Les résultats obtenus par le circuit de capture dépendent en fait de l'activité des glossines, qui elle-même est sous la dépendance des facteurs extrinsèques (climat, nourriture etc.) et des facteurs intrinsèques (sexe, âge, état nutritionnel etc.). Glossina tachinoides ne viendra attaquer le captureur que si l'ensemble de ces conditions d'activité sont réunies. Les quantités capturées dépendent donc de nombreuses variables, dont les effets peuvent cependant être très sérieusement limités, si les captures ont lieu durant une même saison, aux mêmes heures, dans un même gîte, suivant un même circuit. Le nombre capturé est égal au produit de la densité par l'activité; si l'activité reste la même sur une même période, les captures varieront comme la densité. La courbe obtenue étant identique à celle relevée avec la méthode du marquage-recapture, justifie cette conclusion, qui est celle d'autres auteurs (LLOYD Ll.) (18) qui acceptent le circuit de capture comme donnant une mesure relative utile de la densité de population.

Lorsque les conditions écologiques sont très fluctuantes, cette méthode ne peut donner de résultats valables.

Si JACKSON, sur Glossina morsitans, trouve une corrélation sérieuse entre la densité réelle et les données du circuit de capture, GLASGOW et DUFFY (8), GLASGOW et WELCH (9) ne considèrent pas les variations du nombre de glossines capturées comme liées aux variations de la densité réelle des populations de Glossina pallidipes. Les résultats obtenus sur des espèces différentes et dans des conditions d'observation peu comparables, sont certainement à l'origine de ces opinions opposées.

Dans nos conditions d'observation (population isolée, stabilité climatique, constance des méthodes pratiquées), la densité apparente obtenue par le circuit de capture fluctue dans le temps de la même façon que la densité réelle fournie par la méthode du marquagerecapture.

- La méthode du marquage-recapture simple est sensée fournir la densité réelle de population, mais sa validité dépend de certains facteurs exposés précédemment.

Ici, la population n'a pas subi de variations brutales entre le marquage et la recapture; en particulier du fait de son isolement, les fluctuations dues à une émigration ou une immigration éventuelles sont à écarter. Seuls ont joué les facteurs natalité et mortalité, mais non pas de façon brutale.

Le délai de 48 heures entre le marquage et la recapture a été choisi, car il autorise une bonne dispersion et un brassage satisfaisant. C'est lui qui est retenu pour la méthode de capture-marquage simple, appliquée ici. Pour les besoins d'autres observations, des recaptures ont été effectuées aux délais de $6 \mathrm{j}, 9 \mathrm{j}, 13 \mathrm{j}$ et même $16 \mathrm{j}$; les quantités m recapturées alors (tableau I, p. 443) vont en s'amenuisant, suivant une progression sensiblement géométrique et conditionnent $\mathbf{P}$, dont les valeurs deviennent de plus en plus grandes (tableau II, p. 444) et ne peuvent être valablement retenues, car $\mathrm{m}$ recapturée ne représente plus seulement la dispersion des glossines, mais surtout leur disparition par mortalité. On a représenté sur le graphique III (p. 444), les courbes de variations de $P$ (mâles et femelles) lorsque la méthode est appliquée à des délais de recaptures variables, pour la période où le point de lâcher a été toujours le même. On constate qu'aux délais de 2,6 et $9 \mathrm{j}$, les courbes obtenues se superposent très bien; des valeurs de $\mathrm{m}$, enregistrées à ces délais, de façon régulière dans le temps, conduisent à des valeurs de $\mathrm{P}$ quantitativement différentes pour une même date, mais variant de façon identique. Par contre, dès que l'on atteint le 


\begin{tabular}{|c|c|c|c|c|c|c|c|c|c|c|c|c|c|c|c|c|}
\hline $862 \angle 0 T$ & $\angle 06$ IS & $\angle I L$ OZ & $852 \quad 2 \mathrm{I}$ & $90<5$ & $58^{\circ} L$ & $g^{\prime} \varepsilon I$ & $8^{6} \varepsilon z$ & $L^{\prime} 6 E$ & $6^{\prime} E L$ & $=\cdot$ SOW & & & & & & \\
\hline- & $86 \eta \varepsilon I$ & - & $662 L$ & $s \varsigma 9 \varepsilon$ & - & $E I$ & - & $O E$ & $\angle 9$ & - & $6 \angle \varepsilon$ & - & $\varepsilon \angle \nabla$ & 625 & घ97 & $Z L^{\cdot} S^{\circ} \angle I$ \\
\hline- & $\tau \pitchfork 08 \mathrm{I}$ & $290 \mathrm{~L}$ & 6967 & $650 \varepsilon$ & - & $\eta I$ & 07 & 29 & 76 & - & $\varepsilon \angle \downarrow$ & 625 & $\angle 65$ & 727 & $7 \varepsilon s$ & $\tau L^{\circ} 5^{\circ} 0 \mathrm{I}$ \\
\hline $87 t \quad 65$ & $8 \varepsilon L \eta Z$ & - & $\angle 625$ & $998 \varepsilon$ & $\eta$ & SI & - & $S L$ & 86 & 727 & $\$ 99$ & - & $2 \pi L$ & $6 \angle 9$ & 855 & $z L^{\circ} 7^{*} 9 Z$ \\
\hline - & $\angle \forall 0 \mathrm{II}$ & $9 \angle 2<$ & 8669 & $\mathrm{I} 62 \mathrm{~s}$ & - & IE & 27 & IS & $6 L$ & - & 216 & $6 \angle 9$ & $z+L$ & 698 & 187 & でサ・6I \\
\hline T96 ع9 & $\varepsilon 88 \quad 97$ & $\varepsilon O Z E I$ & $\varepsilon 656$ & $8 I 97$ & $\varsigma$ & $\varepsilon \mathrm{I}$ & โ⿷ & $8 \varepsilon$ & $\varepsilon 6$ & $6 \angle 9$ & $2+L$ & 698 & $T L L$ & 216 & $I \angle H$ & $Z \iota^{\circ} \nabla^{\circ} Z I$ \\
\hline$\varsigma 09 \varepsilon \varsigma$ & $19 L 8 \mathrm{~T}$ & $z \ngtr 0 \quad \tau z$ & ZSL SI & $896 \angle$ & 6 & $\subseteq \mathbf{I}$ & $8 \pi$ & $\angle Z$ & $\angle B$ & ZI6 & zES & $9 \tau L$ & 708 & $80 L$ & 625 & $z L^{\cdot} \varepsilon^{\cdot} 6 z$ \\
\hline $698 \mathcal{L} \mathcal{E}$ & $126<7$ & $8 Z 18 Z$ & $256 \varepsilon 2$ & $0 † 26$ & $\varepsilon I$ & 6 & OT & $7 z$ & 59 & $\angle O L$ & $9 \varepsilon L$ & OBt & T86 & $5 Z 0 \mathrm{I}$ & 985 & $Z L^{\circ} \varepsilon^{\circ} \varsigma L$ \\
\hline OZE $\$ 9$ & SO6 $8 E$ & ES† 91 & $068 \varepsilon \mathrm{I}$ & $E O L S$ & $\mathrm{tt}$ & $9 \mathrm{I}$ & SE & 75 & $8 \pi t$ & ५ZO I & 888 & $\varepsilon 28$ & $0 \angle 0 I$ & 096 & IOL & $Z L^{*} E^{*} I$ \\
\hline $6 † \varepsilon \varepsilon 9 \dagger$ & SOZ IOE & $7 \varepsilon \tau$ ( 67 & 87872 & ऽઈЕ $\eta$ & 0 & $z$ & II & $0 z$ & $\angle 6$ & $\varepsilon 28$ & $0 \angle 0 I$ & 096 & 288 & $\angle 7 L$ & દ९ऽ & $Z L \cdot Z \cdot \varepsilon Z$ \\
\hline $082 \quad$ โ8 & 78267 & SOL $L Z$ & $દ 8 Z$ દI & $90<<$ & 9 & 6 & $7 I$ & IZ & It & 096 & 788 & $\angle \eta L$ & $90 L$ & 229 & 805 & $Z L^{\circ} Z^{\prime} 9 \tau$ \\
\hline$\angle 58 \quad 6 £$ & zoz oz & $\angle Z S 9 \mathrm{~T}$ & $6 \angle 68$ & oø\& $L$ & $L$ & $\varepsilon \tau$ & $\mapsto \tau$ & 62 & $\eta E$ & $O S L$ & $90 L$ & 279 & $00 L$ & $0 \angle 9$ & $Z \angle E$ & $z L^{*} z^{\cdot} \cdot 6$ \\
\hline 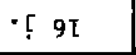 & $\bullet \subsetneq \varepsilon \mathrm{I}$ & • 6 & • 9 & $\cdot[2$ & - $9 \mathrm{I}$ & $\bullet \sqsubset \varepsilon L$ & . 6 & - 9 & - $z$ & • & $\cdot\lceil\varepsilon \mathrm{I}$ & - 6 & • & 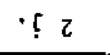 & & \\
\hline \multicolumn{5}{|c|}{ 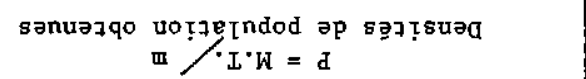 } & \multicolumn{5}{|c|}{ 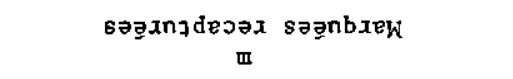 } & \multicolumn{5}{|c|}{$\underset{I}{\text { agnjdesad [RjoI }}$} & $\begin{array}{c}\text { h } \\
\text { sagnbzeUI }\end{array}$ & 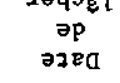 \\
\hline
\end{tabular}


TABLEAU No II

Comparaison des valeurs obtemues pour $\boldsymbol{P}$ à différents délais de recaptures (moyennes)

\begin{tabular}{|c|c|c|c|c|}
\hline$P 2 \mathrm{j}$. & $P 6 \mathrm{j} . / \mathrm{P} 2 \mathrm{j}$. & P 9 j./P $2 j$. & P $13 \mathrm{j} . / \mathrm{P}_{2} \mathrm{j}$. & $P 16 \mathrm{j} . / \mathrm{P} 2 \mathrm{j}$. \\
\hline 1 & 2,14 & 3,63 & 9,09 & 18,80 \\
\hline
\end{tabular}

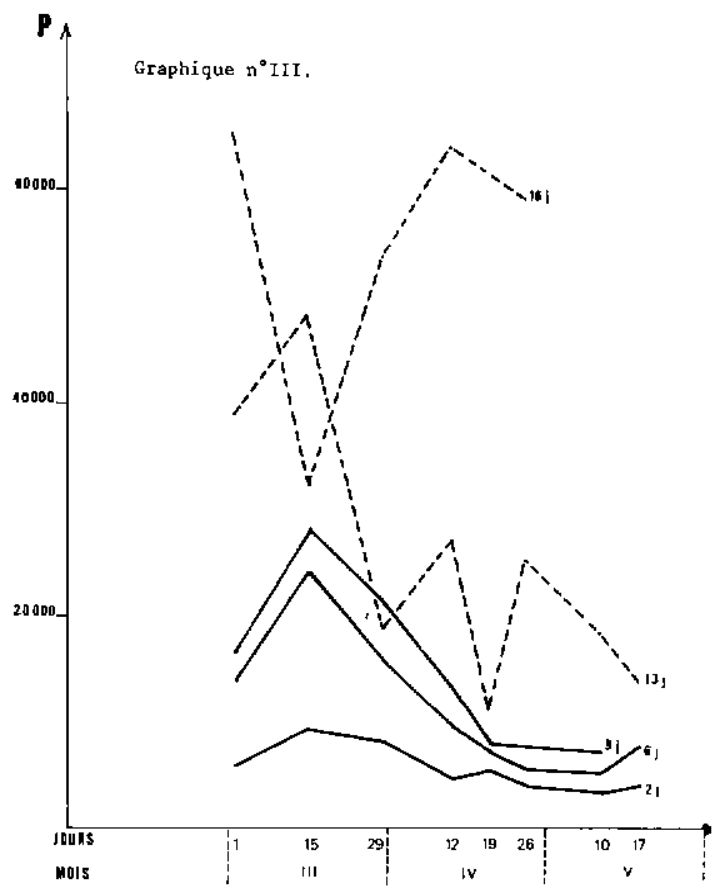

Graph. $\Pi^{\circ}$ III. - Evolution de la densité de population suivant les délais utilisés avec la méthode de marquage-recapture $(\mathbf{P}=\mathrm{M} . \mathrm{T} . / \mathrm{m})$.

délai de $13 \mathrm{j}$ et plus encore celui de $16 \mathrm{j}$, les courbes deviennent aberrantes car $\mathrm{m}$ est alors trop petit.

Autrement dit, le délai de 48 heures semble donner une bonne appréciation de la valeur quantitative réelle de la population. Des délais plus longs $(6 \mathrm{j}, 9 \mathrm{j})$ donnent une valeur relative; mais celle-ci permet de suivre l'évolution graphique de la population dans le temps.

La taille de $\mathrm{m}$ conditionne l'évaluation correcte de la densité effective de la population; elle dépend du délai de recapture, mais aussi du lieu de lâcher de $M$. L'idéal serait de lâcher les glossines marquées en des points multiples pour leur assurer une bonne dispersion. Dans l'observation, tous les lâchers ont eu lieu au même point (01), sauf le 16 et le 23 février (point 02), période pour laquelle $\mathrm{m}$ a fluctué anormalement.
- La méthode du circuit de capture fournit donc une densité apparente, tandis que celle du marquage-recapture donne une densité réelle. La première méthode permet la capture d'une partie de la population : la fraction active qui vient attaquer le captureur; elle est fonction de la « disponibilité » ou availability des auteurs anglo-saxons, ou disposition qu'ont les glossines à venir piquer.

Dans un lieu donné et pour une saison déterminée, on peut calculer le rapport qui existe entre la fraction de population prise sur un circuit de capture et la population totale existante.

Cette aptitude à piquer dépend des facteurs extrinsèques et intrinsèques déjà cités. Lorsque les conditions climatiques d'activité sont optimales pour $G$. tachinoides, on capture en saison chaude environ 70 p. 100 de mâles. Selon BURSELL (19), les mâles sont capturés lorsqu'ils sont en phase 2 ou 4 de la digestion, alors que les femelles sont prises en phase 4 seulement, ce qui explique cette faible proportion des femelles dans les captures manuelles. On écartera donc les femelles de l'estimation de l'availability. Considérant les mâles seuls, on a calculé le rapport de la densité réelle à la densité apparente (tableau III) pour le gîte défini précédemment et pour la durée de la saison chaude 1972 . Il varie pratiquement de 5 à 10 et sa valeur moyenne se situe à 6,4 . Il y a environ 6 mâles dans le gîte lorsqu'on en capture un sur le circuit de capture.

A titre de comparaison, le tableau IV donne les valeurs de ce rapport pour d'autres espèces de glossines en Afrique de l'Est (in GLASGOW) (7).

Donc, la méthode du circuit de capture a l'avantage de la simplicité; sa valeur est relative. Le nombre de glossines prises par heure et par captureur donne un point de repère précieux, si le protocole de capture reste toujours le même dans un gîte bien défini et pendant une durée de temps dont les caractéristiques climatiques varient peu. 
TABL $N^{\bullet}$ III-Evaluation de la "disponibilité" he G. taohinoidea dans les conditiona d'observation

\begin{tabular}{|c|c|c|c|}
\hline Dates & $\begin{array}{c}\text { Dengité réelle } \\
(\mathrm{P}=\mathrm{M} . \mathrm{T})\end{array}$ & $\begin{array}{c}\text { Densité apparente } \\
\text { (circuit de capture) }\end{array}$ & $\begin{array}{c}\text { Rapport ("disponibilité" } \\
\text { ou "availability") }\end{array}$ \\
\hline 9.2 .72 & 4188 & 441 & 10,18 \\
\hline 16.2 .72 & 3992 & 440 & 9,07 \\
\hline 23.2 .72 & 2528 & 482 & 5,24 \\
\hline 1.3 .72 & 3167 & 608 & 5,20 \\
\hline 15.3 .72 & 4350 & 610 & 7,13 \\
\hline 29.3 .72 & 3802 & 453 & 8,39 \\
\hline 12.4 .72 & 2111 & 426 & 4,95 \\
\hline 19.4 .72 & 2539 & 502 & 5,05 \\
\hline 26.4 .72 & 1978 & 398 & 4,96 \\
\hline 10.4 .72 & 1522 & 335 & 4,54 \\
\hline 17.5 .72 & 1630 & 290 & 5,62 \\
\hline
\end{tabular}

TABL. $N^{\bullet}$ IV-Valeurs de 1a "disponibilité" pour différentes espèces de glossines

\begin{tabular}{|c|c|c|c|}
\hline E s p è ce & Pays & $\begin{array}{l}\text { "Disponibilité" } \\
\text { ("availability") }\end{array}$ & Auteurs \\
\hline G. m. morsitans & $\begin{array}{l}\text { Tanzanie } \\
\text { (Kakoma) }\end{array}$ & 7,5 & $\begin{array}{l}\text { Jacks on } \\
\text { (1944) }\end{array}$ \\
\hline G. m. morsitans & $\begin{array}{l}\text { Tanzanie } \\
\text { (Gedamara) }\end{array}$ & $11,2-7,4$ & $\begin{array}{l}\text { Jackson } \\
(1953)\end{array}$ \\
\hline G. swynnertoni & $\begin{array}{l}\text { Tanzanie } \\
\text { (Gedamara) }\end{array}$ & $15,1-7,2$ & $\begin{array}{l}\text { Jackson } \\
\text { (1953) }\end{array}$ \\
\hline G. morsitans & $\begin{array}{l}\text { Ouganda } \\
\text { (Ankolé) }\end{array}$ & $526-50$ & $\begin{array}{l}\text { Har1ey } \\
(195 B)\end{array}$ \\
\hline G. f. fuscipes & $\begin{array}{c}\text { Kenya } \\
\text { (Port Victoria) }\end{array}$ & 176 & $\begin{array}{l}\text { Glasgow } \\
(1954)\end{array}$ \\
\hline G. f. fuscipes & $\begin{array}{l}\text { Kenya } \\
\text { (Kuja River) }\end{array}$ & 88 & $\begin{array}{l}\text { Glasgow } \\
(1954)\end{array}$ \\
\hline G. f. fuscipes & $\begin{array}{l}\text { Kenya } \\
\text { (Sari River) }\end{array}$ & 150 & $\begin{array}{l}\text { Glasgow } \\
\text { (1954) }\end{array}$ \\
\hline G. pallidipes & $\begin{array}{c}\text { Kenya } \\
\text { (Lambwe) }\end{array}$ & $1000-200$ & $\begin{array}{c}\text { Johns in Jackson } \\
(1954)\end{array}$ \\
\hline G. tachinoides & $\begin{array}{l}\text { Tchad } \\
\text { (N'Djamena) }\end{array}$ & $10,1-4,5$ & $\begin{array}{l}\text { Cuisance } \\
(1972)\end{array}$ \\
\hline
\end{tabular}

Dans de telles conditions, elle a permis de tracer une courbe de densité apparente identique à celle de la densité réelle obtenue par marquage-recapture; cette dernière méthode, simple dans son principe, exige pour son application un échantillonnage important, un brassage satisfaisant et une population qui ne subit pas de changements brusques.

\section{LA METHODE DU MARQUAGE-RECAPTURE SIMPLE ET LES METHODES DES MARQUAGES-RECAPTURES REPETES (METHODES DE JACKSON)}

La méthode de marquage-recapture simple est celle employée précédemment; après un 
seul lâcher de glossines marquées, une seule capture a lieu au bout de 48 heures; elle permet de reprendre un certain nombre de mouches marquées et d'aboutir à une estimation de population basée donc sur les résultats d'un seul sondage «marquage-recapture »:

$$
\left(P=\frac{\text { M.T. }}{\mathrm{m}}\right)
$$

Au délai de 48 heures choisi, on suppose que la proportion des glossines marquées aux glossines non marquées est le reflet d'une bonne dilution et que la fraction marquée lâchée $M$ n'a subi aucune décroissance due à la mortalité. En fait, lorsque les facteurs écologiques ne varient pas ou très peu, cette proportion de glossines marquées décroît d'une façon régulière qui suit sensiblement une progression géométrique (voir § précédent). Si on prend des échantillons à des intervalles de temps réguliers après le marquage, on peut estimer le taux de décroissance de la fraction marquée. Lorsqu'il est établi, il devient possible d'estimer la dilution exacte au jour même du marquage et par conséquent de calculer la valeur de la population à cette date.

JACKSON (13) a proposé deux méthodes basées sur ce principe (méthode " positive " et méthode "négative "); elles font appel à des marquages ou à des recaptures répétés; les résultats seront comparés à ceux de la méthode de marquage-recapture simple.

\section{METHODE « POSITIVE " DE JACKSON}

$\begin{gathered}\mathrm{M} \rightarrow \\ \text { marqué } \\ \begin{array}{c}\text { en une } \\ \text { seule } \\ \text { occasion }\end{array}\end{gathered}$$\left\{\begin{array}{c}\frac{\mathrm{T} \text { capturé à différ. occasions }}{\mathrm{T}_{1} \mathrm{~T}_{2} \mathrm{~T}_{3} \mathrm{~T}_{4}} \\ \frac{\mathrm{m} \text { recapturé à différ. occasions }}{\mathrm{m}_{1} \mathrm{~m}_{2} \mathrm{~m}_{3} \mathrm{~m}_{4}}\end{array}\right.$

\section{A. Protocole expérimental}

Il consiste à lâcher en une seule occasion une quantité $\mathbf{M}$ (assez grande) de mouches marquées. On pratique après ce lâcher plusieurs recaptures successives au cours desquelles on enregistre le nombre $\mathrm{m}$ de glossines marquées. Celles-ci sont relâchées après enregistrement de leurs quantités.
Un lâcher a lieu par semaine. Les recaptures se font suivant un circuit qui reste toujours le même. Elles ont été limitées à 4 séances aux délais de $2,6,9$ et $13 \mathrm{j}$ après le lâcher. Les techniques de marquage sont celles déjà décrites.

Pour pouvoir comparer les résultats, les quantités marquées et celles recapturées sont ramenées à 100 individus. Pour chaque délai de recapture, on calcule la valeur corrigée suivante :

$$
\begin{aligned}
& \mathrm{y}_{1}=\frac{\mathrm{m}_{1} \times 100 \times 100}{\mathrm{M} . \mathrm{T}_{1}} \\
& \mathrm{y}_{2}=\frac{\mathrm{m}_{2} \times 100 \times 100}{\mathrm{M} . \mathrm{T}_{2}}, \text { etc. }
\end{aligned}
$$

On obtient ainsi $\mathrm{y}_{1}, \mathrm{y}_{2}, \mathrm{y}_{3}, \mathrm{y}_{4}$ et l'on recherohe $y_{0}$. Il faut donc trouver le rapport moyen de chaque valeur de y à la valeur qui la précède et en particulier de $\mathrm{y}_{1}$ à $\mathrm{y}_{0}$.

$$
\begin{gathered}
r=\frac{y_{2}+y_{3}+y_{4}}{y_{1}+y_{2}+y_{3}} \\
\text { et } y_{0}=\frac{y_{1}+\ldots+y_{k-1}}{r}\left(y_{1} \ldots y_{k-2}\right)
\end{gathered}
$$

Ayant le point d'extrapolation $y_{0}$, on a la population : $\mathbf{P}=\frac{10^{4}}{\mathrm{y}_{0}}$.

\section{B. Résultats}

La méthode "positive" de JACKSON a été appliquée à la population globale (mâles et femelles) d'une part, et à celle des mâles seuls, d'autre part.

- Appliquée aux quantités de glossines lâchées puis recapturées successivement sans tenir compte des sexes, la méthode donne, pour la période d'observation considérée, une courbe évolutive de la population représentée sur le graphique IV; après une chute brutale, on observe un accroissement de densité qui atteint son maximum le 29 mars pour décroître dans les jours suivants. Comparée à la courbe obtenue avec la méthode de marquage-recapture simple, on constate une similitude d'aspect avec cependant un maximum situé 15 jours plus tard dans le temps.

Les valeurs comparées de densité fournies par les deux méthodes donne en moyenne une population totale de 5706 individus ( \pm 1236 ) 


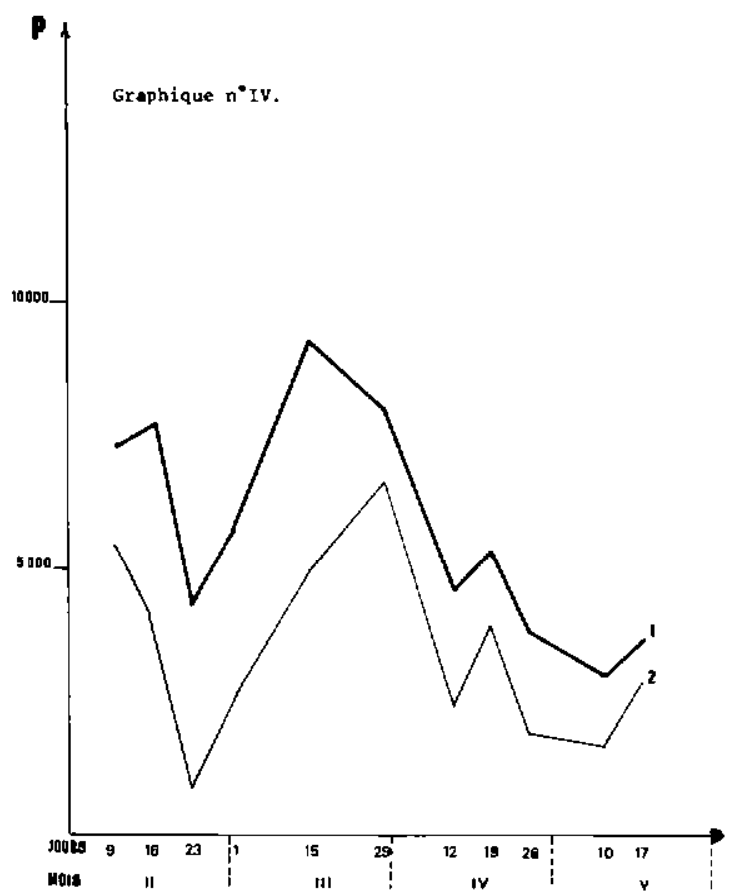

Graph. no IV. - Evolution de la densité de population (mâles et femelles) suivant la méthode du marquage-recapture simple (1) et la méthode « positive " de Jackson (2).

pour le marquage-recapture simple et 3301 \pm 1087 pour la méthode «positive» de JACKSON.

- Considérons maintenant les courbes de densité de population fournies par les deux méthodes en ne retenant que la population mâle (graphique V). Les mêmes remarques s'imposent : les deux courbes ont même aspect, mais les maximums atteints sont décalés de 15 jours.

La méthode de marquage-recapture simple indique une population de 2891 mâles ( \pm 635 ) tandis que celle de JACKSON en accuse seulement 1662 ( \pm 590).

\section{Discussion}

S'il s'agit de savoir comment la population fluctue, les deux méthodes donnent des résultats sensiblement identiques, car les courbes ont même aspect. Si on veut chiffrer exactement les fluctuations de densité, les données enregistrées diffèrent significativement entre les deux méthodes $(t=2,92$ pour la population globale; $t=2,83$ pour la population mâle).

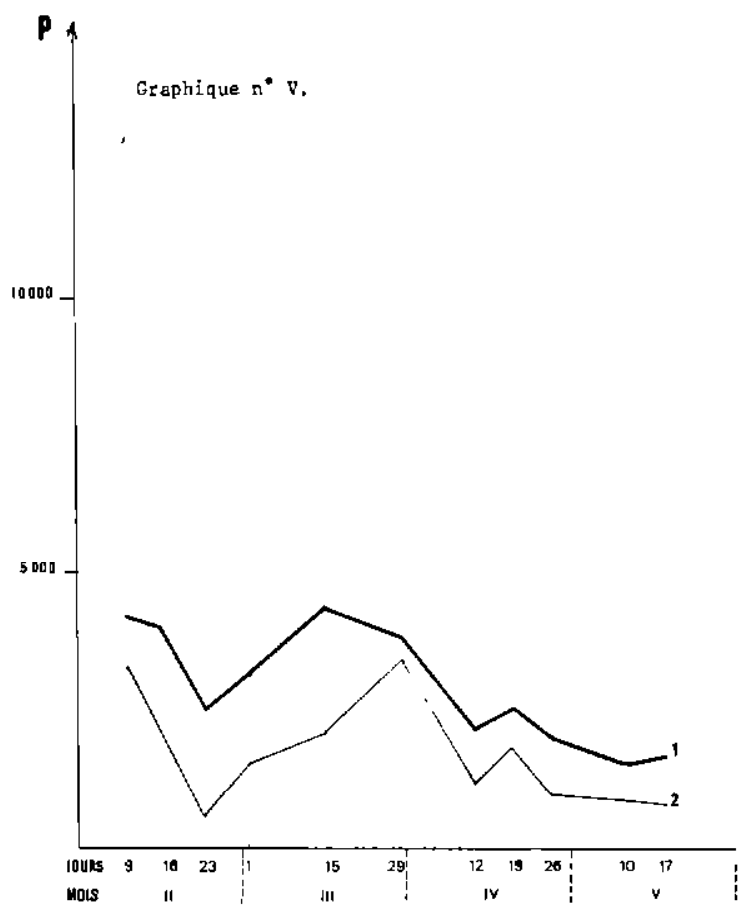

Graph. no V. - Evolution de la densité de population (mâles seulement) suivant la méthode du marquage-recapture simple (1) et la méthode " positive » de Jackson (2).

La vraie valeur de la population totale se situe entre 5706 et 3301 et celle des mâles seuls entre 2891 et 1662 .

Du fait d'un échantillonnage unique à un délai rapproché (48 heures), la première méthode surévalue quelque peu la population réelle.

Les résultats de la deuxième méthode faisant appel à différents délais de recapture semblent approcher plus certainement de la densité réelle; toutefois, certains de ces délais étant importants $(9 \mathrm{j}, 13 \mathrm{j})$ la valeur de $\mathrm{P}$ est vraisemblablement sous-estimée.

\section{METHODE « NEGATIVE " DE JACKSON}

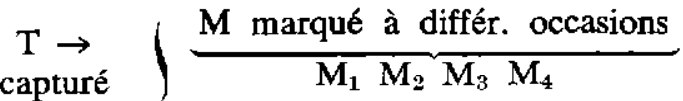

$$
\begin{aligned}
& \begin{array}{l}
\text { en une } \\
\text { occasion }
\end{array} \underbrace{m_{2}}_{m_{1}} m_{3} m_{4} \text { recapturé en une seule occas. }
\end{aligned}
$$

\section{A. Protocole expérimental}

La méthode consiste à effectuer des marquages au cours de plusieurs occasions successives 
(une couleur par jour de capture); lors de la recapture unique qui les suit, on enregistre alors les glossines d'après la couleur du marquage qu'elles portent.

On procède donc de façon inverse à la méthode "positive ". L'observation se situe sur la même durée de temps : la saison chaude.

Les marquages ont été limités au nombre de quatre, espacés entre eux d'environ une semaine (quelques-uns quinze jours); la recapture finale a donc lieu environ trois semaines après le premier marquage.

De la même façon que pour la méthode précédente, on a corrigé les nombres obtenus comme si 100 mouches avaient été marquées et 100 mouches recapturées. On obtient alors les valeurs $\mathrm{y}_{1}, \mathrm{y}_{2}, \mathrm{y}_{3}, \mathrm{y}_{4}$ et on calcule le rapport moyen r qui permettra de trouver $\mathrm{y}_{0}: \mathrm{P}=\frac{10^{4}}{\mathrm{y}_{0}}$

\section{B. Résultats}

La courbe ainsi obtenue pour la saison considérée (graphique VI) traduit un accroissement de la densité de population totale (mâles et femelles) jusqu'à la mi-mars, suivi d'une chute progressive jusqu'au mois de mai. $\mathrm{Si}$, pour la même période, on la compare à la courbe tracée avec les résultats de la méthode de marquage-recapture simple, on constate une similitude : elles ont en commun un aspect de courbe en clocher, le maximum étant atteint à la même date.

Par contre, les valeurs calculées de densité obtenues par les deux méthodes sont différentes $(t=6,79)$. Si la population du gîte est estimée à 5706 individus d'un côté, elle n'est que de 1475 土 375 par la méthode "négative».

Considérant la population de mâles seuls, les mêmes remarques s'imposent: les courbes de densité suivent la même évolution, atteignent leurs maximums à la même date, mais les populations estimées sont quantitativement différentes :

$$
2891 \pm 635 \text { et } 656 \pm 99(t=5,53)
$$

\section{Discussion}

La méthode "négative" permet de savoir si la population croît ou décroît et concorde avec la méthode de marquage-recapture simple.

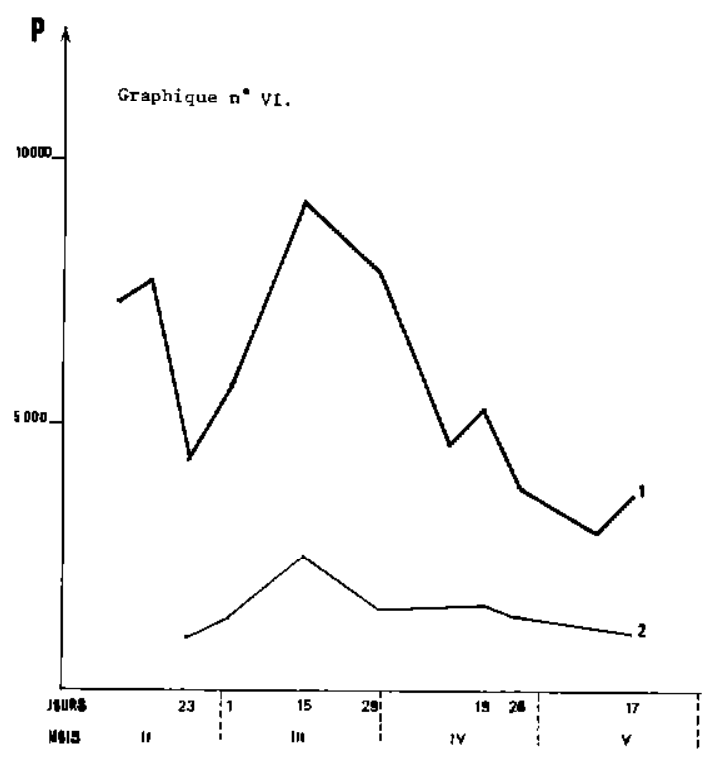

Graph. no VI. - Evolution de la densité de population (mâles et femelles) suivant la méthode du marquage-recapture simple (1) et la méthode «négative» de Jackson (2).

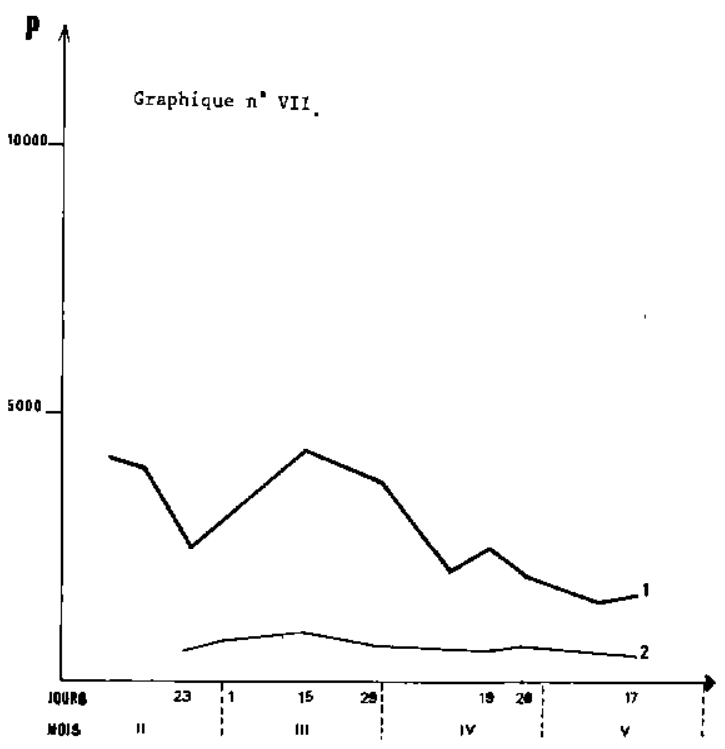

Graph. no VII. -- Evolution de Ia densité de population (mâles seulement) suivant la méthode de marquage-recapture simple et la méthode "négative » de Jackson.

Elle nous donne cependant ici une mauvaise estimation de la densité réelle.

La méthode de marquage-recapture simple basée sur un seul délai de courte durée $(2 \mathrm{j})$ donne une valeur de la densité légèrement surestimée. La méthode "négative " appliquée ici avec des délais de marquage trop grands, sousestime certainement la population. 
JACKSON trouvait des valeurs très semblables entre les résultats de ses deux méthodes ("positive" et "négative") sur une même période d'observation. En fait, les résultats devraient être très proches, si, entre les recaptures successives de la méthode "positive " et entre les marquages successifs de la méthode " négative ", les délais étaient les mêmes. Pour les besoins de différents objectifs visés dans cette expérience, nos délais de recaptures ont été de 2, 6, 9 et $13 \mathrm{j}$, alors que ceux des marquages étaient de 3 semaines, 2 semaines, 1 semaine avant la recapture finale. Les valeurs de $\mathrm{m}$ recapturé, différentes, expliquent ces écarts.

\section{viII. CONCLUSION}

L'observation a porté sur une population de Glossina tachinoides isolée dans l'espace et concentrée sur une faible surface, autorisant ainsi des captures numériquement importantes; elle s'est déroulée sur une période climatique relativement stable (saison chaude).

- D'un point de vue qualitatif, la méthode du circuit de capture et celles du marquagerecapture simple ou répété, fournissent des données très concordantes quant à l'évolution de la densité de cette population. Croissance et décroissance se situent aux mêmes moments et le maximum a lieu dans tous les cas entre le 15 et le 30 mars.

- D'un point de vue quantitatif, seules les méthodes de marquage-recapture permettent une approche de l'évaluation de la densité réelle; leur principe est simple, mais elles exigent d'être appliquées de façon constante et régulière. Le point de lâcher en particulier restera toujours le même.

Les méthodes "positive» et «négative 》 de JACKSON doivent donner sensiblement les mêmes résultats, si les délais de recaptures de l'une et les délais de marquages de l'autre sont identiques et assez courts. Reposant sur plusieurs délais, les valeurs de densité obtenues sont certainement plus proches de la réalité, mais ces méthodes faisant appel à des captures répétées, sont longues et astreignantes.

$\mathrm{Si}$ les méthodes de marquage-recapture sont seules à donner des valeurs numériques de la population $\mathbf{P}$, leur application n'est possible que dans des gîtes de densité forte ou moyenne; lorsque celle-ci est faible, les quantités $M, T$ et $\mathrm{m}$ sont trop basses pour donner des résultats valables. La méthode du circuit de capture peut alors rendre de grands services; en particulier, pour apprécier la valeur d'une méthode de lutte, elle permettra de suivre la décroissance de densité d'une population réduite et de juger des dernières étapes de son extermination.

\section{SUMMARY}

\section{Compared density estimate of an isolated population of Glossina tachinordes West by two common methods: the fly-round and marking-recapture techniques}

Methods for evaluating the estimated density (fly-round technique) and absolute density (simple or replicated marking-recapture techniques) were used simultaneously in a Glossina tachinoides habitat during the dry season, a rather stable climatic period in which Glossina concentrate in a habitat bordering on the Chari River.

Under observation conditions, the qualitative evolution of the density curves of the population thus isolated is the same for the two techniques.

From a quantitative point of view, absolute density can be evaluated only by marking-recapture techniques; the approach of this density depends on the regular application of these techniques, the delays in marking or recaptures; large populations are also required so that the techniques may be valid. The fly-round technique can then supersede them and be very useful.

\section{RESUMEN}

\section{Apreciación comparada de la densidad de una población aislada de Glossina tachinoides West. mediante dos métodos clásicos: el circuito de captura y los marcados - nuevas capturas}

Se aplicaron simultáneamente métodos de evaluación de la densidad aparente (método del «circuito de captura» o afly-round») y de la 
densidad real (marcado-nueva captura simple o repetido) en un sitio de descanso de Glossina tachinoides durante la estación seca, periodo climático relativamente estable, durante la cual las glosinas se concentran en un sitio de las orillas del Chari.

En las condiciones de la observación, la evolución cualitativa de las curvas de densidad de la población asi aislada es semejante con los dos métodos.

Cuantitativamente, solos los métodos de marcado-nueva captura permiten una evaluación de la densidad real.

La determinación de ésta mediante dichos métodos depiende de su regularidad de aplicación, de los plazos de marcados o de nuevas capturas; Necesitan tambien poblaciones numericamente importantes bajo pena de perder su validez. Entonces se puede substituir el método del «circuito de captura " pudiendo hacer grandes servicios.

\section{BIBLIOGRAPHIE}

1. ANDREWARTHA (H. G.), BIRCH (L. C.). The distribution and abondance of animals. University of Chicago Press, 1954, XVI, 782 p.

2. BAILEY (N.T.J.). On estimating the size of mobile populations from recapture data. Biometrika, 1951, 38 : 293-306.

3. BAILEY (N. T. J.). Improvements in the interpretation of recapture data. Biometrika, 1952, 28: 120-127.

4. BUXTON (P.A.). The natural history of tsetse flies. London, H. K. Lewis and Co Ltd, 1955, 816 p.

5. CUISANCE (D.), ITARD (J.). Comportement de mâles stériles de Glossina tachinoides W. lâchés dans les conditions naturelles. Environs de Fort-Lamy (Tchad). II. Longévité, dispersion. Rev. Elev. Méd. vét. Pays trop., 1973, 26 (2): 169-186.

6. DAVIES (H.). Tsetse flies in Northern Nigeria. Ibadan University Press, 1967, 268 p.

7. GLASGOW (J.P.). The distribution and abundance of tsetse. Oxford, Pergamon Press, 1963, $252 \mathrm{p}$.

8. GLASGOW (J. P.), DUFFY (B. J.). Traps in field studies of Glossina pallidipes Austen. Bull. ent. Res., 1961, 52: 795.

9. GLASGOW (J. P.), WELCH (J. R.). Long-term fluctuations in numbers of the tsetse fly Glossina swynnertoni Austen. Bull. ent. Res., 1962, $53: 129$.

10. GRUVEL (J.). Contribution à l'étude écologique de $G$. rachinoides dans la réserve de Kalamaloué, vallée du Bas-Chari. Thèse Doct. Sci., Paris. 1974.

11. JACKSON (C. H. N.). On a method of marking tsetse flies. J. Anim. Ecol., 1933, 2: 238-246.
12. JACKSON (C. H. N.). Some new methods in the study of G. morsitans. Proc. Zool. Soc. Lond., $1936,4: 812-96$

13. JACKSON (C. H.N.). The analysis of an animal population. J. anim. Ecol, 1939, 8: 238-246.

14. JACKSON (C. H. N.). The analysis of a tsetse fly population II. Ann. Eugen., Lond., 1942, 11 : 176-205.

15. LE BERRE (R.). Cours du D.E.A. d'Ecologie. Faculté des Sciences de Paris, 1972.

16. LESLIE (P. H.), CHITTY (D.). The estimation of population parameters from data obtained by means of the capture-recapture method. I. The maximum likelihood equation for estimating the dearth-rate. Biometrika, 1951, 38: 269-92.

17. LINCOLN (F. C.). Calculating waterfowl abundance on the basis of banding returns, Circ. U.S. Dep. Agric., 1930 n $^{\circ} 118$.

18. LLOYD (L.L.). Assessment of a tsetse population. Bull. ent. Res., 1936, 27 : 261-267.

19. MULLIGAN (H.W.). The african trypanosomiases. London, George Allen and Unwin Ltd., $1970,950 \mathrm{p}$.

20. POTTS (W. H.). A contribution to the study of numbers of tsetse fly (Glossina morsitans Westw.) by quantitative methods. S. Afr. J. Sci., 1930, 27 : 491-497.

21. Rapports annuels, Région de Recherches Vétérinaires et Zootechniques d'Afrique Centrale, Laboratoire de Farcha, 1966 à 1970.

22. SADELER (C. B.). Cours du D.E.A. d'Ecologie. Faculté des Sciences de Paris, 1971.

23. TIBAYRENC (R.), ITARD (J.), CUISANCE (D.). Marquage des glossines par des poudres fluorescentes, Rev. Elev. Méd. vét. Pays trop., 1971,24 (2) : 277-286. 